EPJ manuscript No.

(will be inserted by the editor)

\title{
Creating loffe-Pritchard micro-traps from permanent magnetic film with in-plane magnetization
}

\author{
I. Barb, R. Gerritsma, Y. T. Xing, J. B. Goedkoop, and R. J. C. Spreeuw \\ Van der Waals - Zeeman Institute, University of Amsterdam, Valckenierstraat 65, 1018 XE Amsterdam, The Netherlands \\ e-mail: ibarb@science.uva.nl
}

the date of receipt and acceptance should be inserted later

\begin{abstract}
We present designs for Ioffe-Pritchard type magnetic traps using planar patterns of hard magnetic material. Two samples with different pattern designs were produced by spark erosion of $40 \mu \mathrm{m}$ thick FePt foil. The pattern on the first sample yields calculated axial and radial trap frequencies of $51 \mathrm{~Hz}$ and $6.8 \mathrm{kHz}$, respectively. For the second sample the calculated frequencies are $34 \mathrm{~Hz}$ and $11 \mathrm{kHz}$. The structures were used successfully as a magneto-optical trap for ${ }^{87} \mathrm{Rb}$ and loaded as a magnetic trap. A third design, based on lithographically patterned $250 \mathrm{~nm}$ thick FePt film on a Si substrate, yields an array of 19 traps with calculated axial and radial trap frequencies of $1.5 \mathrm{kHz}$ and $110 \mathrm{kHz}$, respectively.
\end{abstract}

Key words. atom chips - magnetic trapping - cold atoms

PACS. 03.75.Be Atom and neutron optics - 39.25.+k Atom manipulation (scanning probe microscopy, laser cooling, etc.) - 75.70.-i magnetic properties of thin films, surfaces, and interfaces

\section{Introduction}

Planar microstructures have recently emerged as a very powerful and attractive tool for handling neutral ultracold atoms. These so-called "atom chips" have been used to construct miniature atom optical elements including traps, waveguides, and beamsplitters [1,2,3,4,5]. Atom chips are now in development to miniaturize atom optics and to make it more robust. They have great potential for application in quantum information processing $6,7,8,8$ and in atom interferometry. Large magnetic field gradients and thus large trapping forces can be achieved on-chip, removing the necessity of large, high power external coils. Miniature current carrying wires can produce tight mag- 
netic trapping potentials that have successfully been used to create Bose-Einstein condensates of rubidium atoms 9 , 10.

Most atom chips so far have relied on a pattern of current-carrying wires to generate the required magnetic field gradients. Here we investigate a promising alternative based on patterned hard magnetic films. Waveguide designs using out-of-plane magnetized film have been reported before using TbFeCo [1] and CoCr films [12,13 and magneto-optically patterned $\mathrm{Co} / \mathrm{Pt}$ thin films [14. In plane magnetized videotape has been used for waveguides and traps 15] 16. Here we describe the fabrication of selfbiased Ioffe-Pritchard microtraps based on FePt film.

The use of permanently magnetized film has many potential benefits, including very large magnetic field gradients $\left(\sim 10^{4} \mathrm{~T} / \mathrm{m}\right)$. Even for very intricate patterns there is no ohmic power dissipation, no current noise from power supplies, and no stray field from lead wires. It is quite conceivable that up to $10^{5}$ micro traps may be integrated on a square centimeter. An obvious advantage of currentconducting chips is the possibility to switch or modulate the currents. As an alternative, we use external uniform magnetic fields to manipulate the magnetic field minima.

In this paper we investigate the possibilities of microscopic patterns of permanent magnetization, in-plane magnetized. In the second section we briefly discuss the requirements on the magnetic materials, motivating our choice of FePt alloy. Section 3 is dedicated to analysis of the patterns that were prepared and discussions of their application on atom chips. We describe two types of hard magnetic atom chips. Our trap designs, together with the actually produced samples, show that in-plane magnetization is as suitable as out-of-plane magnetization for the purpose of creating (arrays of) traps. The first design, on a scale of $100 \mu \mathrm{m}$, was cut by spark erosion out of $40 \mu \mathrm{m}$ thick FePt foil. The second design, an array of strips on a scale of $1 \mu \mathrm{m}$, was lithographically patterned in a $250 \mathrm{~nm}$ thick film of FePt on Si.

\section{Material properties}

The material to be used in permanent-magnetic atom chips must meet a number of requirements. In order to produce a sufficiently large stray field, a large remanent magnetization $\mathrm{M}_{r}$ is required. Because we use external fields ( $\leq$ $10 \mathrm{mT}$ ) to move field minima around we also need a large enough coercivity. Finally, a high magneto-crystalline anisotropy will ensure that the magnetization is preserved regardless of the shape of the structure. We found that FePt meets these criteria. FePt has been studied extensively both in bulk [17] and thin-film [18] form since it combines high magneto-crystalline anisotropy with high saturation magnetization $\mathrm{M}_{s}[19$ and has excellent stability and corrosion resistance. At high temperature FePt has a disordered face-centered cubic (fcc) structure which has a very high $\mathrm{M}_{s}$ but is magnetically soft. The lowtemperature equilibrium structure on the other hand is face-centered tetragonal (fct or $\mathrm{L}_{0}$ ), with a lower $\mathrm{M}_{s}$ but very high magneto-crystalline anisotropy and coercivity. In this phase the $\mathrm{Fe}$ and $\mathrm{Pt}$ order in an atomic multilayer structure. It has been shown [20] that annealing of soft fcc 
material obtained from a melt produces nanocrystallites of

the fct phase that are exchange coupled to the soft phase,

resulting in a material with the optimum properties desired here. FePt keeps its magnetic properties for different thicknesses, both as a bulk and as a film. A unique feature of the FePt material is that it has a nearly isotropic and large coercivity, making it suitable for both in-plane and out of plane field orientation. The former is suitable for the creation of tight traps, while the latter is suitable for creating waveguides.

We prepared two types of samples. The first consists of a pattern cut by spark erosion out of bulk $\mathrm{Fe}_{0.6} \mathrm{Pt}_{0.4}$ foil. The measured ratio $\mathrm{M}_{r} / \mathrm{M}_{s}$ is $\sim 0.8$, the saturation magnetization $\mathrm{M}_{s} \approx 400 \mathrm{kA} / \mathrm{m}$ and the coercivity is about 0.2 $\mathrm{T}$, which is enough to withstand the highest external field that is applied to manipulate the atoms $(10 \mathrm{mT})$. Tests showed that baking at $170^{\circ} \mathrm{C}$, to obtain UHV conditions, does not affect the magnetic properties.

The second type of sample consists of an array of strips in 250-nm thick $\mathrm{Fe}_{0.5} \mathrm{Pt}_{0.5}$ film on $\mathrm{Si}$ using lithographic techniques. For this film thickness the magnetic properties are essentially similar to the bulk material. The film has been grown by Molecular Beam Epitaxy (MBE). The inplane remanence, saturated magnetization and coercivity of the film are: $\mathrm{M}_{r} / \mathrm{M}_{s}=0.90, \mathrm{M}_{s} \approx 600 \mathrm{kA} / \mathrm{m}$ and $\mathrm{H}_{c} / \mu_{0}$ $=0.80 \mathrm{~T}$. More details on the material optimization and lithographic processing are given in separate publications 21,22].

\section{Designing loffe Pritchard traps}

\subsection{General remarks}

Magnetic traps of the Ioffe-Pritchard (IP) type $23,24,25$ 26. have been used extensively in the realization of BoseEinstein condensation of alkali gases. A crucial property of these traps is that they have a non-zero magnetic field minimum, in order to prevent Majorana transitions to untrapped magnetic sublevels. The basic configuration for an IP trap consists of four long bars with currents in alternating directions. This generates a cylindrical quadrupole field, which leads to radial confinement. A set of axial coils is used to produce a non-zero axial field and to pinch off the trap along the axis. Atoms in a weak-field seeking spin state are trapped in the minimum of the magnetic field magnitude.

This basic layout can be implemented with permanent magnets to create a self biasing structure. Two parallel magnetic strips produce a cylindrical quadrupole field in a similar way to the four current carrying bars in a IP trap. The axial field including the pinch fields are added by placing extra pieces of material in appropriate places. All dimensions can be scaled down $(<100 \mathrm{~nm})$, resulting in large gradients and curvatures.

The designs described below aim at achieving a trap depth of at least $0.5 \mathrm{mT}$, trap frequencies greater than $1 \mathrm{kHz}$ and a non-zero minimum field so as to avoid spin flipping. The stray field of the patterns has been calculated using Mathematica 27 in combination with the Radia package [28]. 


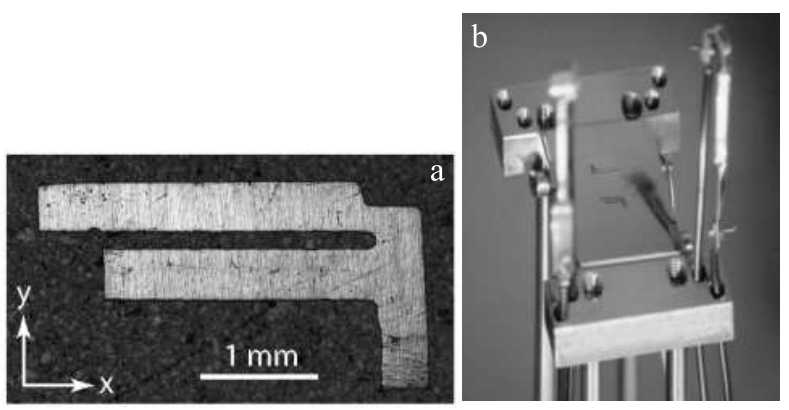

Fig. 1. (a): Ioffe-Pritchard trap cut out of a $40 \mu \mathrm{m}$ thick FePt foil, magnetized in-plane (\|y). (b): Two similar foil traps glued on an aluminum mirror for mounting inside the vacuum system. The lower structure corresponds to the left picture.

\section{2 loffe Pritchard trap based on FePt foil}

A cylindrical quadrupole field can be easily created in the vicinity of a magnetic pattern, using two identical, uniformly magnetized strips. The trap is so designed that the two long strips, in-plane magnetized, produce a cylindrical quadrupole field with its axis along the $x$ direction, see Fig. [1 The bias field needed to lift the magnetic field minimum is given by the structure itself, in this particular case by the stem of the "F" and the extension of the upper long strip. These two parts also determine the trap depth. The structure is thus a self-biased IP trap. This particular shape is motivated partly by practical considerations while cutting, and by the desire to have a single connected piece to simplify mounting on the mirror surface.

We have glued two such magnetic structures with slightly different dimensions on an aluminum mirror shown in Fig. 1(b). The ultra-high vacuum compatible glue should be used with restraint, since excess glue will cause undesired scattering of light and hamper absorption imaging of trapped atoms. The surface of the FePt foil also causes some diffuse light scattering, which is why it appears somewhat darker than the mirror in Fig. 1(b). The surface area of the F structures is sufficiently small that it does not affect the operation of a mirror magneto-optical trap (MMOT). The resulting magnetic field pattern produced by one of the structures, Fig. 1(a), is shown in Fig. 2 The field strength as a function of the distance to the surface is shown in Fig. 21a). The magnetic field contour lines in the $x y$ plane are shown in Fig. 2(b), marking the B-field strength in multiples of $0.5 \mathrm{mT}$. A local field minimum of $0.25 \mathrm{mT}$ is found at a height $z=0.19$ $\mathrm{mm}$ above the surface. Both figures 2(a) and 2(b) are cuts through the position of minimum field. The calculated axial and radial trap frequencies, for ${ }^{87} \mathrm{Rb}$ in the $\mathrm{F}=\mathrm{m}_{f}=$ 2 state, are $51 \mathrm{~Hz}$ and $6.8 \mathrm{kHz}$, respectively and the trap depth is about $1.1 \mathrm{mT}(760 \mu \mathrm{K})$. The second structure, upper in Fig. 1(b) forms a trap of $34 \mathrm{~Hz}$ in the axial direction and $11 \mathrm{kHz}$ in the radial direction with a trap depth of $3.3 \mathrm{mT}(2.3 \mathrm{mK})$.

The magnetic foil was prepared from bulk nanocrystalline $\mathrm{Fe}_{0.6} \mathrm{Pt}_{0.4}$ material. The material was rolled to a $100 \mu \mathrm{m}$ foil and mechanically polished to $40 \mu \mathrm{m}$ thickness. Spark erosion using a $50 \mu \mathrm{m}$ wire was used to cut the FePt foil. The size of the gap between the two strips is determined by the diameter of the cutting wire.

The mirror was mounted inside the vacuum cell with the glued magnetic structures facing downward. We have trapped $2 \times 10^{6}{ }^{87} \mathrm{Rb}$ atoms in a mirror magneto optical trap (MMOT) using the field gradient of the magnetic structures and an external field of $0.2 \mathrm{mT}$ along $y$. The 

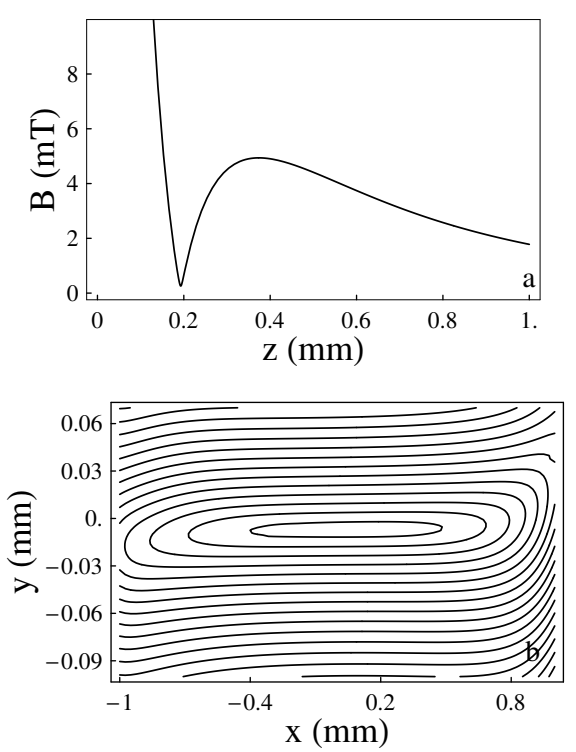

Fig. 2. Calculated magnetic field magnitude above the F structure. (a). Field magnitude versus distance from the chip surface. The minimum is $0.25 \mathrm{mT}$. (b) Magnetic field contour lines of the self-biased IP magnetic trap in the $x y$ plane, in multiples of $0.5 \mathrm{mT}$.

MMOT forms $\sim 2 \mathrm{~mm}$ under the chip. This proves that the sample is still magnetized after baking of the vacuum setup. Closer to the structure the magnetic field gradient becomes so strong that the atoms can be magnetically trapped. We have trapped $5 \times 10^{5}$ atoms in this IP trap using an external field of $6 \mathrm{mT}$. An absorption image of the atomic cloud at $400 \mu \mathrm{m}$ under the chip is shown in Fig. 3 The next step will be to switch off the external field so that the atoms will be trapped in the self biased IP trap, requiring detailed optimization of the trajectory into the bias free trap.

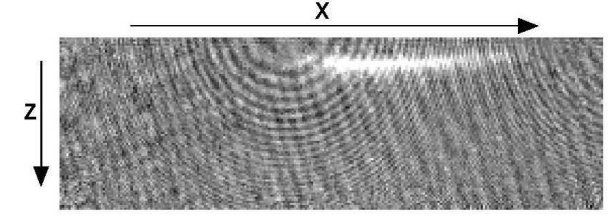

Fig. 3. An atomic cloud magnetically trapped in the magnetic field gradient of the structure, $400 \mu \mathrm{m}$ under the chip surface using an external field of $6 \mathrm{mT}$ in the $\mathrm{x}$ direction. The field of view is $3.3 \times 1.3 \mathrm{~mm}^{2}$.

\subsection{Arrays of traps based on micro-fabricated FePt thin films}

In a second approach we designed arrays of magnetic strips creating arrays of magnetic traps. Such arrays should enable one to move atoms from one trap to another as in a shift register practical for quantum information processing. This purpose requires traps on the micrometer scale, implying films with (sub-)micrometer thickness. Calculations show that a thickness of $250 \mathrm{~nm}$ is satisfactory for making sufficiently tight magnetic trapping potentials. Each array is characterized by the length and width of the strips, the width of the slit between strips, and the longitudinal displacement of neighboring strips as can be seen in Fig. 固 By keeping the thickness of the magnetic material constant and varying the other parameters we can achieve different trapping potentials at different heights above the surface with the possibility to reach frequencies in the range of $10-100 \mathrm{kHz}$. Higher frequencies, in the $\mathrm{MHz}$ range could be achieved by further reduction of the dimensions.

The working principle of this design can be explained as follows: (n-1) cylindrical quadrupoles are formed be- 
tween $\mathrm{n}$ equally long strips. As one can see neighboring strips are shifted with respect to each other by a constant step, like a staircase (see Fig.4(a)). As a result, for every pair of neighboring strips the upper strip extends a little to the right of the pair, while the lower strip extends to the left. These extending pieces of magnetic material pinch off the ends of each trap and produce the axial field. This results in a trap with nonzero field minimum being formed between each pair of neighboring strips, in this case 19 traps using 20 strips. We observe that the trap depth increases with the increase in the size of these "end caps". One example of magnetic field patterns above the film are presented in Fig. 目 A plot of the magnetic field contours reveals a periodic array of magnetic field minima as shown in Fig 4(d). The trap frequencies calculated for the central trap are $110 \mathrm{kHz}$ in the radial direction and $1.5 \mathrm{kHz}$ in the axial direction. The traps are $1.4 \mathrm{mT}(980$ $\mu \mathrm{K})$ deep. The traps are formed at $4 \mu \mathrm{m}$ from the surface.

The patterning has been done by e-beam lithography and plasma etching. Fig. 1 s shows SEM images of an array of these patterns and a cross section of one of them. This shows that the etching process has yielded an edge at approximately $45^{\circ}$ angle, with a roughness of approximately $50 \mathrm{~nm}$, which is on the order of the nanocrystalline grain size. This edge roughness is slightly larger than the state of the art in current-conducting chips and can probably still be improved. The edge roughness has recently been shown to be a critical feature for creating waveguides in current-conducting chips. An analysis of the requirements for permanent-magnet chips is in progress. For both types
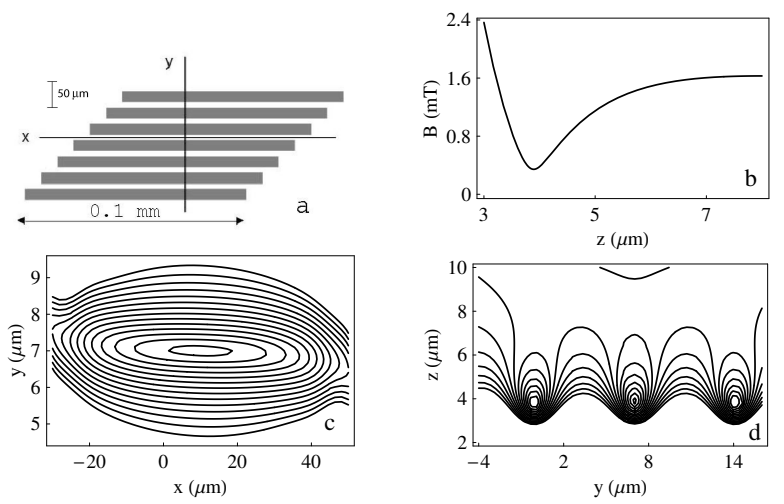

Fig. 4. Calculated array of micro-traps above the surface. (a) Each strip has a width of $5 \mu \mathrm{m}$ and a length of $100 \mu \mathrm{m}$. The slit is $2 \mu \mathrm{m}$ and the longitudinal (x) shift is $10 \mu \mathrm{m}$. (b) Field strength versus $z$ through the center of the trap. (c) Contours of constant field strength in the $x y$ plane. The magnetic field contour lines are at $0.3 \mathrm{mT}$ intervals. (d) Contours of magnetic field magnitude in a $y z$ plane showing an array of magnetic traps.

of chips the requirements for creating traps should be less stringent, depending on the aspect ratio of the traps. The final chip will be coated with a thin Pt layer to obtain a reflecting surface allowing the formation of the MMOT, and so the photoresist remaining on top of the FePt pattern will have no effect.

\section{Discussion and conclusions}

We have shown that self-biased micron sized Ioffe-Pritchard traps and arrays of traps can be prepared with permanent magnetic structures with in-plane magnetization. We have presented two types of structures using FePt as magnetic material and two types of chips have been prepared using foil (40 $\mu \mathrm{m}$ thickness) and films (250 nm thickness), with diverse geometries. The bulk structure and the ar- 

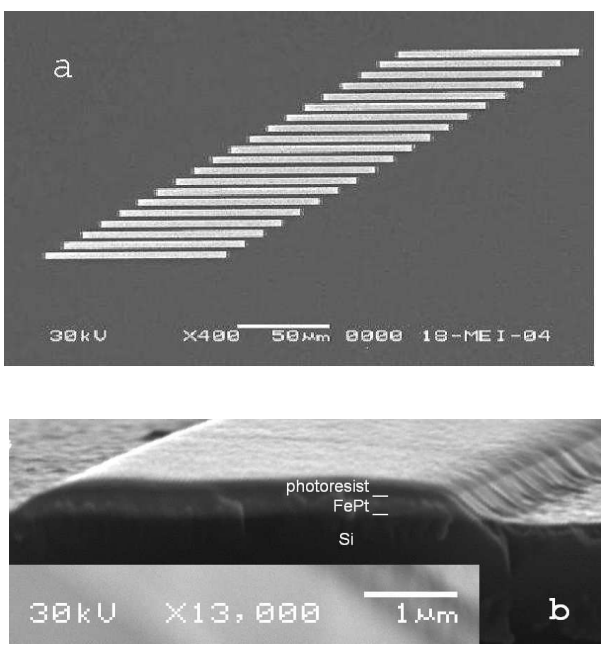

Fig. 5. (a)An array of strips of FePt on a silicon substrate and (b)cross section of one of the FePt strips.

rays of strips are suitable for producing neutral atom traps with high frequencies. The strong magnetization of FePt in combination with the small structures produce large magnetic field gradients of order $10^{4} \mathrm{~T} / \mathrm{m}$. The FePt film can be patterned down to nm scales, giving extremely tight traps with $100 \mathrm{kHz}$, possibly $\mathrm{MHz}$, frequencies for $\mathrm{Rb}$ atoms, and making this method promising for quantum information processing. Previous theoretical [29] and experimental $30,31,32$ results have shown that at short distances of several $10 \mu \mathrm{m}$ to the surface the lifetime can be reduced by thermally-induced spin relaxation. The spin flip lifetime depends on the film thickness, atom-surface distance and the skin depth 33,34]. For our films of 250 nm thickness, with an estimated skin depth $\delta=100 \mu \mathrm{m}$ at 2.1 $\mathrm{MHz}$ it should be possible to create long lived traps at $\gtrsim 2 \mu \mathrm{m}$ distance from the surface. The large and nearisotropic coercivity of FePt makes it suitable for both inplane and out of plane magnetization. These microstructures with permanent-magnetic films offer a large flexibil- ity in design and length scales ranging from mm to (sub-) $\mu \mathrm{m}$ feature sizes. A highly promising future approach will be to develop hybrid chips, combining magnetic structures with other techniques, including electrostatic potentials, optics, etc.

We thank H. Schlatter, H. Luigjes and C. Rétif for expert technical assistance and support. This work was made possible by the fabrication and characterization facilities of the Amsterdam nanoCenter. This work is part of the research program of the Stichting voor Fundamenteel Onderzoek van de Materie (Foundation for the Fundamental Research on Matter) and was made possible by financial support from the Nederlandse Organisatie voor Wetenschappelijk Onderzoek (Netherlands Organization for the Advancement of Research). This work was supported by the EU under contract MRTN-CT-2003-505032.

\section{References}

1. R. Folman et al., Phys. Rev. Lett. 84, 4749 (2000).

2. J. Reichel, W. Hänsel, and T. Hänsch, Phys. Rev. Lett. 83, 3398 (1999).

3. R. Folman et al., Adv. At. Mol. Opt. Phys. 48, 263 (2002).

4. J. Reichel, Appl. Phys. B 75, 469 (2002).

5. N. Dekker et al., Phys. Rev. Lett. 84, 1124 (2000).

6. P. Treutlein et al., Phys. Rev. Lett. 92, 203005 (2004).

7. T. Calarco et al., Phys. Rev. A 61, 022304 (2000).

8. E. Jané et al., Quant. Inf. \& Comp. 3, 15 (2003).

9. W. Hänsel, P. Hommelhoff, T. W. Hänsch, and J. Reichel, Nature 413, 498 (2001).

10. H. Ott et al., Phys. Rev. Lett. 87, 230401 (2001). 
11. A. Sidorov et al., C. R. Acad. Sci. Paris, Série IV 2, 565 (2001).

12. T. J. Davis, J. Opt. B: Quantum Semiclass. Opt. 1, 408 (1999).

13. A. Sidorov et al., Acta Physica Polonica B 33, 2137 (2002).

14. S. Eriksson et al., Appl. Phys. B 79, 811 (2004).

15. P. Rosenbusch et al., Appl. Phys. B 70, 709 (2000).

16. C. D. J. Sinclair et al., arXiv:physics/0502073.

17. Q. Xiao et al., J. All. Comp. 364, 315 (2004).

18. M. Weisheit, L. Schultz, and S. Fähler, J. Appl. Phys. 95, 7489 (2004).

19. D. Weller and M. Doerner, Ann. Rev. Mat. Sc. 30, 611 (2000).

20. J. Liu, C. Luo, Y. Liu, and D. Sellmyer, Appl. Phys. Lett. 72, 483 (1998).

21. Y. T. Xing et al., unpublished .

22. Y. T. Xing et al., Phys. Stat. Sol. (c) 12, 3702 (2004).

23. Y. Gott, M. Ioffe, and V. Tel'kovskii, Nucl. Fusion, 1962 Suppl. Pt. 3, 1045 (1962).

24. D. E. Pritchard, Phys. Rev. Lett. 51, 1336 (1983).

25. T. Bergeman, G. Erez, and H. J. Metcalf, Phys. Rev. A 35, 1535 (1987).

26. V. Bagnato et al., Phys. Rev. Lett. 58, 2194 (1987).

27. Wolfram Research, Inc., Mathematica, Version 5.0 (Champaign, IL, 2003).

28. O. Chubar, P. Elleaume, and J. Chavanne, J. Synchroton Rad. 5, 481 (1998), the Radia code is freely available for download from http://www.esrf.fr/machine/support/ids/Public/Codes/software.html

29. C. Henkel, S. Pötting, and M. Wilkens, Appl. Phys. B 69, 379 (1999).
30. Y. Lin, I. Teper, C. Chin, and V. Vuletić, Phys. Rev. Lett. 92, 050404 (2004).

31. M. P. A. Jones et al., Phys. Rev. Lett. 91, 080401 (2003).

32. D. Harber, J. McGuirk, J. Obrecht, and E. Cornell, J. Low Temp. Phys. 133, 229 (2003).

33. S. Scheel, P. Rekdal, P. Knight, and E. Hinds, arXiv:quant-ph/0501149.

34. P. K. Rekdal, S. Scheel, P. L. Knight, and E. A. Hinds, Phys. Rev. A 70, 013811 (2004). 